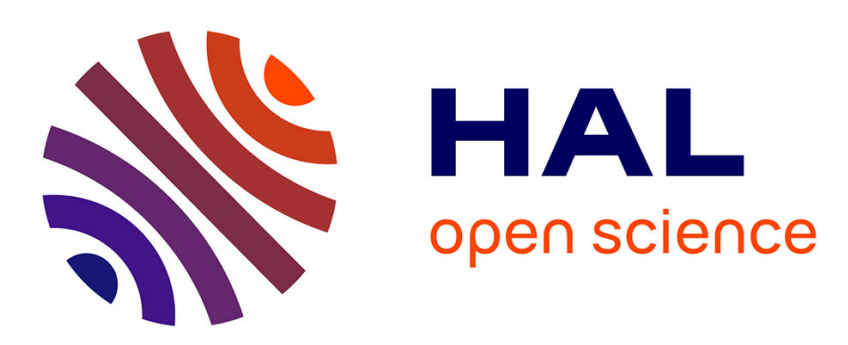

\title{
High order approximation of a tokamak edge plasma transport minimal model with Bohm boundary conditions
}

Sebastian Minjeaud, Richard Pasquetti

\section{- To cite this version:}

Sebastian Minjeaud, Richard Pasquetti. High order approximation of a tokamak edge plasma transport minimal model with Bohm boundary conditions. Journal of Computational Physics, 2015, pp.84-87. hal-01144699

\section{HAL Id: hal-01144699 \\ https://hal.univ-cotedazur.fr/hal-01144699}

Submitted on 20 Oct 2016

HAL is a multi-disciplinary open access archive for the deposit and dissemination of scientific research documents, whether they are published or not. The documents may come from teaching and research institutions in France or abroad, or from public or private research centers.
L'archive ouverte pluridisciplinaire HAL, est destinée au dépôt et à la diffusion de documents scientifiques de niveau recherche, publiés ou non, émanant des établissements d'enseignement et de recherche français ou étrangers, des laboratoires publics ou privés. 


\title{
NOTE
}

\section{High order approximation of a tokamak edge plasma transport minimal model with Bohm boundary conditions}

\author{
Sébastian Minjeaud and Richard Pasquetti ${ }^{1}$ \\ Lab. J. A. Dieudonné, UMR CNRS 7351, Université de Nice-Sophia Antipolis, \\ F-06108 Nice, France \& INRIA project CASTOR.
}

\begin{abstract}
Keywords:
Magnetic confined fusion; Edge plasma; Bohm boundary conditions; Hyperbolic system; Tokamaks.
\end{abstract}

In the recent paper [2], a penalty method is proposed to address the so-called Bohm boundary conditions which are generally imposed to model the limiters, i.e. the walls that intercept in a tokamak the magnetic field lines. This approach is validated by considering a simple one-dimensional hyperbolic system, that constitutes a minimal transport model for ionic density and momentum. In [1] this penalty model is discussed and an improved formulation of the penalty method is proposed. More recently, in [6], a similar model is considered but completed with evolution equations for the ionic and electronic temperatures. Again, a penalty method is proposed to enforce the boundary conditions at the walls.

In the present note, we consider the system studied in $[1,2]$ and show that as soon as the solution is smooth, a spectrally accurate approximation can be obtained. To this end, a stabilized spectral element method (SEM) is prefered to the Godunov schemes used in $[1,2,6]$. Moreover, instead of the penalty approach, which in the multidimensional context can only offer a first order algebraic convergence, we use a direct imposition of the Bohm boundary condition in an explicit time marching. The system studied in $[1,2]$ writes:

$$
\begin{aligned}
& \partial_{t} N+\partial_{x} \Gamma=S_{N} \\
& \partial_{t} \Gamma+\partial_{x}\left(\frac{\Gamma^{2}}{N}+N\right)=S_{\Gamma}
\end{aligned}
$$

with $t \in \mathbb{R}^{+}$and $x \in \Omega=\left(x_{\min }, x_{\max }\right)$ for the time and space variables and where $N$ and $\Gamma$ stand for the dimensionless ion density and ion momentum, respectively. In this model, the ion and electron temperatures are assumed to be constant, so that when using the perfect gas law, in dimensionless form the pressure is equal to the density. We then recognize the usual conservation equations of mass and momentum, where some source terms $S_{N}$ and $S_{\Gamma}$ are considered. Moreover, the dimensionless velocity equals the Mach number, i.e. $M=\Gamma / N$. It is easy to check that the system (1)-(2) is strictly hyperbolic. In convective form it writes:

$$
\partial_{t}\left(\begin{array}{c}
N \\
\Gamma
\end{array}\right)+\left(\begin{array}{cc}
0 & 1 \\
-M^{2}+1 & 2 M
\end{array}\right) \partial_{x}\left(\begin{array}{c}
N \\
\Gamma
\end{array}\right)=\left(\begin{array}{c}
S_{N} \\
S_{\Gamma}
\end{array}\right)
$$

and so the wave speeds, defined as the eigenvalues of the matrix in (3), equal $\lambda_{ \pm}=M \pm 1$. As well known, the number of boundary conditions to be considered depends on the number of ingoing waves. Thus, at $x_{\max }$ two boundary conditions are needed if $M<-1$, one boundary is needed if $-1<M<1$ and no boundary conditions are needed if $M>1$.

\footnotetext{
${ }^{1}$ Corresponding author. E-mail address: richard.pasquetti@unice.fr
} 
In order to model the limiter by a particles sink, the Bohm boundary condition states that $M\left(x_{\max }\right) \geq 1$ [3]. Note that $M\left(x_{\max }\right)=1$ corresponds to the case where the boundary $x=x_{\max }$ is characteristic, so that it is not clear what should be done. Thus, in [1] it is suggested to slightly modify the boundary condition by imposing $M\left(x_{\max }\right)=1-\eta$ and $M\left(x_{\min }\right)=1+\eta$, with a fixed (small) $\eta>0$ in order to consider a wellposed hyperbolic problem. From our point of view, here this problem is mainly theoretical and probably not relevant as soon as numerical time and space discretizations are involved. Hereafter, we enforce the following Bohm conditions:

$$
M\left(x_{\min }\right) \leq-1 \quad \text { and } \quad M\left(x_{\max }\right) \geq 1
$$

To check this approach in the frame of high order methods, we use in space the SEM, i.e. a high order nodal finite element method such that the approximation space, say $E_{h}$, contains all $C^{0}$ functions whose restriction in each element is a polynomial of degree $p$, see e.g. [4]. In each element the basis functions are Lagrange polynomials based on the Gauss-Lobatto-Legendre (GLL) points and these GLL interpolation points are also used as quadrature points (quadratures are then exact for polynomials of degree $2 p-1$ ). Moreover, since the problem is hyperbolic, shocks may develop and a stabilization technique is required. We use the Spectral Vanishing Viscosity (SVV) technique introduced in $[5,7]$ (see also [8] in the multidimensional context). With $\left(N_{h}, \Gamma_{h}\right)$ for the numerical approximations of $(N, \Gamma)$, we thus consider the following variational formulation:

$$
\begin{aligned}
& \int_{x_{\min }}^{x_{\max }} \partial_{t} N_{h} v_{h} d x+\int_{x_{\min }}^{x_{\max }} \partial_{x} \Gamma_{h} v_{h} d x+V_{N}=\int_{x_{\min }}^{x_{\max }} S_{N h} v_{h} d x, \quad \forall v_{h} \in E_{h}, \\
& \int_{x_{\min }}^{x_{\max }} \partial_{t} \Gamma_{h} v_{h} d x+\int_{x_{\min }}^{x_{\max }} \partial_{x}\left(\frac{\Gamma_{h}^{2}}{N_{h}}+N_{h}\right) v_{h} d x+V_{\Gamma}=\int_{x_{\min }}^{x_{\max }} S_{\Gamma h} v_{h} d x, \quad \forall v_{h} \in E_{h}
\end{aligned}
$$

where the SVV stabilization terms write:

$$
V_{N}=\epsilon_{h p} \int_{x_{\min }}^{x_{\max }} Q_{p}\left(\partial_{x} N_{h}\right) \partial_{x} v_{h} d x, \quad V_{\Gamma}=\epsilon_{h p} \int_{x_{\min }}^{x_{\max }} Q_{p}\left(\partial_{x} \Gamma_{h}\right) \partial_{x} v_{h} d x
$$

In these expressions, $\epsilon_{h p}$ controls the amplitude of the SVV term and $Q_{p}$ is the so-called SVV operator whose kernel contains all low frequency Legendre components, say for $k \leq m_{p}<p$ of the spectral approximation, see e.g. [5] for details. In the computations, we have systematically used $\epsilon_{h p}=h / p$, where $h$ is the grid-size, and $m_{p}=[\sqrt{p}]$ ([.], for nearest integer).

For the integration in time, we use a standard fourth order Runge Kutta scheme (RK4 scheme). Then, to enforce the Bohm conditions we impose at the end of each RK4 step:

$$
N_{h}\left(x_{\min }\right):=-\Gamma_{h}\left(x_{\min }\right) \quad \text { if } \quad \frac{\Gamma_{h}}{N_{h}}\left(x_{\min }\right)>-1 \quad ; \quad N_{h}\left(x_{\max }\right):=\Gamma_{h}\left(x_{\max }\right) \quad \text { if } \quad \frac{\Gamma_{h}}{N_{h}}\left(x_{\max }\right)<1 .
$$

On the basis of this SEM approximation, we have considered three different test cases.

Test case 1: This test-case is the one introduced in [2]. The length of the domain is unitary, $\Omega=(0,1)$. At the initial time $t=0$ the fluid is at rest, $M_{0}=\Gamma_{0}=0$, and the density is constant, $N_{0}=1$. For the source terms one has $S_{N}=2$ and $S_{\Gamma}=0$. In [2] the asymptotic solution, i.e. obtained at $t \gg 1$, is compared to the analytical one, say $\left(N_{\infty}, \Gamma_{\infty}\right)$, such that:

$$
N_{\infty}=S_{N}(0.5+\sqrt{x(1-x)}) \quad, \quad \Gamma_{\infty}=S_{N}(x-0.5) .
$$

The computation has been carried out with $K=20$ elements and with the polynomial degree $p=6$. In Fig. 1, we show the solution $\left(N_{h}, \Gamma_{h} / N_{h}\right)$ at different times. At the final time of the computation, the numerical solution clearly agrees with the analytical one. Despite the use of the SVV technique, one can discern that the solution is not perfectly smooth. This is mainly associated to the fact that for this test case the initial profiles $N_{0}$ and $\Gamma_{0}$ do not verify the Bohm conditions, since $M(0)=M(1)=0$ at $t=0$.

Test case 2: This test-case is similar to test-case 1, i.e. $S_{N}=2$ and $S_{\Gamma}=0$, but to check our algorithm 

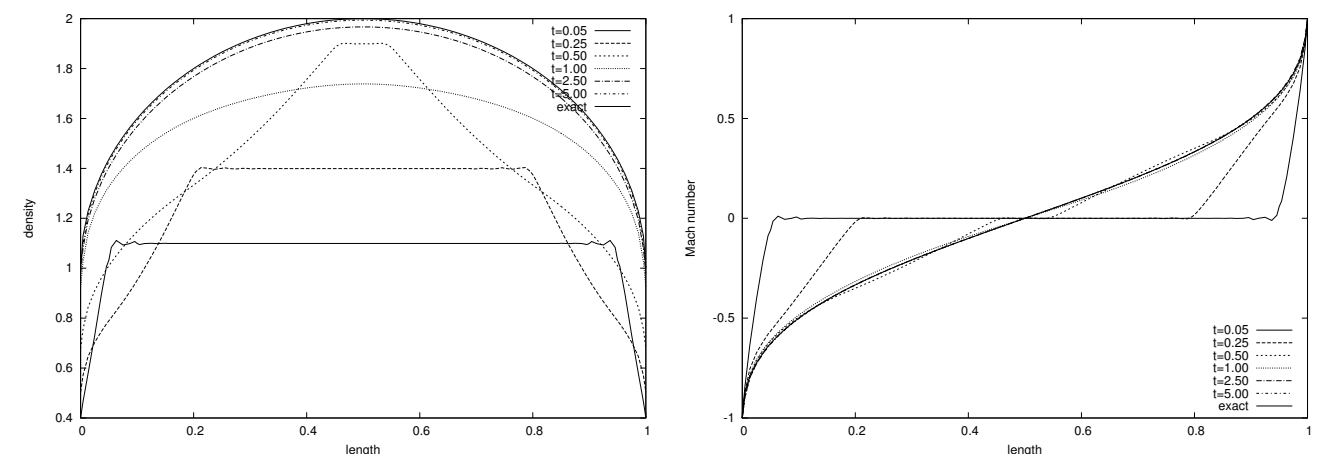

Figure 1: Density and Mach number at different times and exact asymptotic solution. Computation done with $K=20$ elements and a polynomial degree $p=6$. SVV stabilization: $m_{p}=2$ and $\epsilon_{h p}=1 / 120$.

with a supersonic - subsonic transition at the boundary, the initial condition for the momentum is now $\Gamma_{0}=2(x-0.5)-2 \sin (\pi x)$. Then, at the initial time, the flow is sonic at the boundaries and partially subsonic and supersonic inside the domain. This situation may be met in tokamaks [3]. Using again $K=20$ and $p=6$, the solution thus obtained is shown at different times in Fig. 2, where we focus on the shortest times, when occurs the transition. Later on, the evolution is similar to the test-case 1.
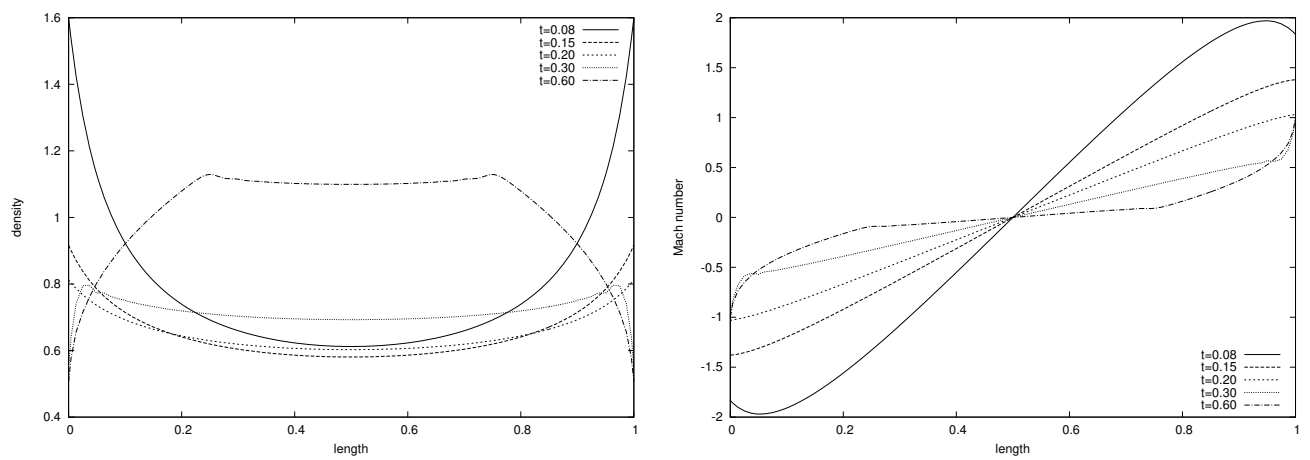

Figure 2: Density and Mach number at different times. Computation done with $K=20$ elements and a polynomial degree $p=6$. SVV stabilization: $m_{p}=2$ and $\epsilon_{h p}=1 / 120$.

It should be mentionned that:

- for the present computation the SVV stabilization is not mandatory. This was not the case previously, with $p=6$, probably because at $t=0,|M( \pm 1)|<1$ for test 1 .

- we have also obtained satisfactory results by enforcing the Bohm conditions at the end of each RK4 step, using:

$$
\Gamma_{h}\left(x_{\min }\right):=-N_{h}\left(x_{\min }\right) \quad \text { if } \quad \frac{\Gamma_{h}}{N_{h}}\left(x_{\min }\right)>-1 \quad ; \quad \Gamma_{h}\left(x_{\max }\right):=N_{h}\left(x_{\max }\right) \quad \text { if } \quad \frac{\Gamma_{h}}{N_{h}}\left(x_{\max }\right)<1,
$$

which points out that in the $1 \mathrm{D}$ case, there is no predominant role of one of the two variables. With $p=6$ this was however not true for the test 1 . Thus, for robustness reasons we prefer to use (8) in the RK scheme.

- Using a more complex initial condition, e.g. $\left(N_{0}=1, \Gamma_{0}=-\cos (3 \pi x)\right)$, one can observe shocks. Such a non physical situation is well supported by the SVV stabilization, even if as expected one observes the Gibbs phenomenon.

Test case 3: This test-case is essentially the one proposed in [1]; For $x \in \Omega=(-0.4,0.4)$

$$
N(x, t)=\exp \left(\frac{-x^{2}}{0.16(t+1)}\right), \quad \Gamma(x, t)=c \sin \left(\frac{\pi x}{0.8}\right)\left(\frac{-x^{2}}{0.16(t+1)}\right)
$$


with $c=1$ in [1]. The source terms $S_{N}$ and $S_{\Gamma}$ are those obtained by plugging these expressions in the governing equations. The initial profiles $N_{0}$ and $\Gamma_{0}$ are those obtained for $t=0$. Clearly, $M=\Gamma / N$ is constant in time and shows a sinusoidal behaviour in space such that $M( \pm 0.4)= \pm 1$ if $c=1$. Such a very smooth solution is of interest to carry out a convergence study. We have introduced the parameter $c$ in order to check our SEM approximation in the case $c>1$, i.e. for a supersonic outgoing flow.
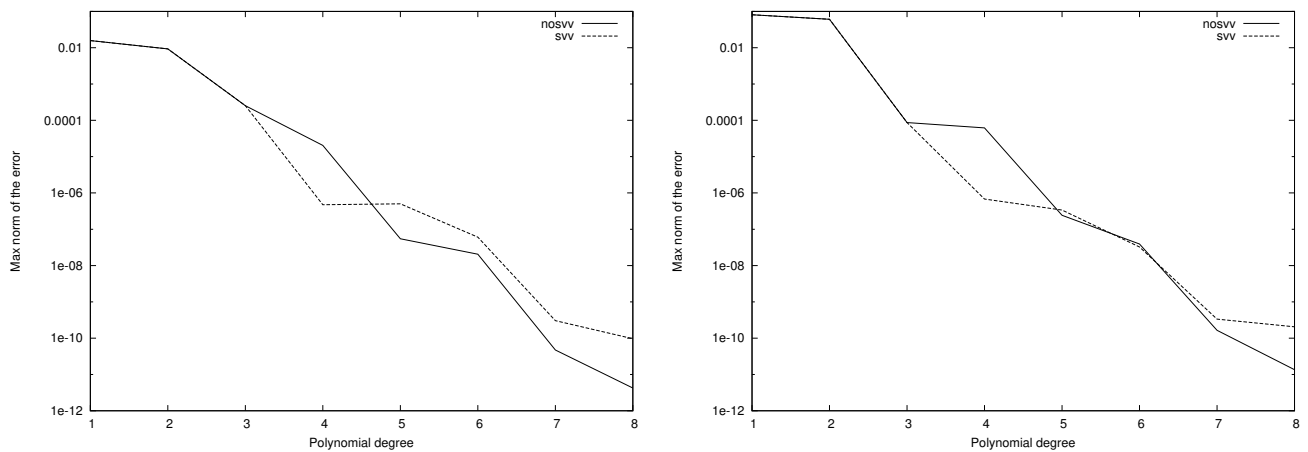

Figure 3: Error versus the polynomial degree with and without SVV stabilization, for the sonic (at left) and supersonic (at right) flows. The space discretization is based on $K=20$ elements. If SVV is used, then $\epsilon_{h p}=0.8 /(20 p)$ and $m_{p}=[\sqrt{p}]$.

The computations were carried out with $K=20$ elements and various polynomial degrees, $1 \leq p \leq 8$. The time-step is chosen small $\left(\Delta t=10^{-4}\right)$ so that the time approximation errors are negligible. Fig. 3 shows the variation of the error, in the max norm (i.e. in the $l^{\infty}$ norm), at $t=1$, with respect to the polynomial degree, for the sonic $(c=1)$ and supersonic flows $(c=2)$, with and without using the SVV stabilization technique. Even if such convergence curves are not really smooth, one clearly discerns the expected SEM exponential rate of convergence.

Acknowledgments: This study was supported by the FR-FCM, project "Fluid numerical modelling of edge plasma of tokamaks. Application to ITER" and by the EUROFUSION, project "Synergetic numericalexperimental approach to fundamental aspects of turbulent transport in the tokamak edge". We also thank all partners of the ANR project "ESPOIR" for fruitful discussions.

\section{References}

[1] Ph. Angot, Th. Auphan, O. Guès, An optimal penalty method for a hyperbolic system modeling the edge plasma transport in a tokamak, J. Comput. Phys. 261 (2014) 1-22.

[2] L. Isoardi, G. Chiavassa, G. Ciraolo, P. Haldenwang, E. Serre, Ph. Ghendrih, Y. Sarazin, F. Schwander, P. Tamain, Penalization modeling of a limiter in the tokamak edge plasma, J. Comput. Phys. 229 (2010) $2220-2235$.

[3] Ph. Ghendrih, K. Bodi, H. Bufferand, G. Chiavassa, G. Ciraolo, N. Fedorczak, L. Isoardi, A. Parades, Y. Sarazin, E. Serre, F. Schwander, P. Tamain, Transition to supersonic flows in the edge plasma, Plasma Phys. Control Fusion 53 (5) (2011) 054019 .

[4] G.E. Karniadakis, S.J. Sherwin, Spectral hp element methods for CFD, Oxford Univ. Press, London, 1999.

[5] Y. Maday, S.M.O. Kaber, E. Tadmor, Legendre pseudo-spectral viscosity method for nonlinear conservation laws, SIAM J. Numer. Anal. 30 (2) (1993) 321-342.

[6] A. Paredes, H. Bufferand, G. Ciraolo, F. Schwander, E. Serre, Ph. Ghendrih, P. Tamain, A penalization technique to model plasma facing components in a tokamak with temperature variations, J. Comput. Phys. 274 (2014) $283-298$.

[7] E. Tadmor, Convergence of spectral methods for nonlinear conservation laws, SIAM J. Numer. Anal. 26 (1) (1989) $30-44$.

[8] C.J. Xu, R. Pasquetti, Stabilized spectral element computations of high Reynolds number incompressible flows, J. Comput. Phys. 196 (2) (2004) 680-704. 